QUIPUIRAMAYOC Revista de la Facultad de Ciencias Contables

Vol. 20 N. ${ }^{\circ} 37$ pp. 106-122 (2012) UNMSM, Lima, Perú

ISSN: 1560-9103 (versión impresa) / ISSN: 1609-8196 (versión electrónica)

\title{
¿ES COMPETITIVA LA INDUSTRIA CEMENTERA PERUANA?
}

\author{
IS COMPETITIVE INDUSTRY PERUVIAN CEMENT? \\ Raúl Arrarte Mera* \\ Docente Asociado de la Facultad de Ciencias Contables - UNMSM
}

[Recepción: Julio de 2012 / Conformidad: Agosto de 2012]

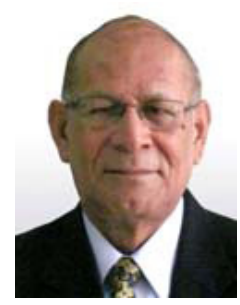

\section{RESUMEN}

El presente estudio se enmarca dentro de la investigación aplicada en base al análisis de los Estados Financieros Comparados, al 31 de Diciembre del 2011-2010, publicados por la Bolsa de Valores de Lima, Perú.

El sector cementero es actualmente el más destacado en la economía nacional, impulsada por la actividad privada, motor de la expansión del sector, por construcción de viviendas multifamiliares, centros comerciales, supermercados, hoteles, y la mayor inversión pública, que incluye la construcción y rehabilitación de carreteras, autopistas, hospitales, colegios, entre otras obras en desarrollo y ejecución.

El análisis practicado es documental-descriptivo-explicativa a partir de los estados financieros publicados e informes de empresas calificadoras de riesgo.

Los objetivos planteados al desarrollar este trabajo, son:

a) Analizar si la industria cementera en el Perú, es competitiva y contribuye a la reducción de costos.

b) Verificar si la industria cementera se benefició con el auge de construcción de viviendas mejorando su productividad.

c) Conocer si la medida de desempeño de valor económico agregado en el sector cementero fue positivo en los años corrientes de 2010 y 2011. El sector es competitivo porque no incrementó el precio unitario de la bolsa de cemento pese al crecimiento de consumo y se benefició con el auge de construcción de viviendas. El valor económico agregado, a una TEA ${ }^{1}$ de $7 \%$, en moneda nacional, fue positiva para todas las empresas cementeras, excepto Cemento Andino SAA. Instamos la promoción de accio-

\section{ABSTRACT}

This study is part of applied research based on analysis of Comparative Financial Statements at December 31,2011 - 2010, published by the Lima Stock Exchange, Peru.

The cement sector is currently the most prominent on the national economy, driven by the private sector, the engine of the expansion of the sector, multifamily housing, shopping malls, supermarkets, hotels, and increased public investment, including construction and rehabilitation of roads, highways, hospitals, schools and other works in development and implementation

The analysis is performed descriptive-explanatory documentary from published financial statements and reports of local rating agencies. The objectives in developing this work are:

a) To examine whether the cement industry in Peru, is competitive and contributes to cost reduction.

b) Check if the cement industry benefited from the housing boom improving productivity.

c) To determine whether performance measure of economic value added in the cement sector was positive in the current years 2010 and 2011. The industry is competitive because it has increased the unit price of the stock despite the growth of cement consumption; benefited from the housing boom. The economic value added, a TEA ${ }^{1}$ of $7 \%$, in local currency, was positive for all cement companies except Cemento Andino SAA. We urge shareholders to promote a widespread approach STATEUNIVERSITY-ENTERPRISE seeking greater productivity with high added value.

* Doctor en Ciencias Contables y Empresariales. Maestro en Administración. Contador Público Colegiado. Docente investigador de la UNMSM. E-mail: raularrarte@speedy.com.pe

1 TEA = Tasa efectiva anual. 
nariado difundido en un enfoque de ESTADOUNIVERSIDAD-EMPRESA buscando mayor productividad con alto valor agregado.

Palabras Clave: Competitividad. estructura de inversión y financiamiento, valor económico agregado.

\section{MARCO TEÓRICO}

\section{INDUSTRIA CEMENTERA PERUANA}

Un reporte sectorial de 15 páginas, procedente del Banco Wiese Sudameris (hoy Scotiabank) ${ }^{2}$, pese a su antigüedad de siete años, nos dice:

- "La industria presenta diversas ventajas competitivas, entre las que destacan barreras naturales a la entrada, tales como mercados geográficos definidos y disponibilidad de materias primas, así como el bajo costo de insumos y el alto poder de negociación frente a clientes y proveedores".

- "La industria mantiene un adecuado nivel de inversiones y actualización tecnológica, y parte de su estrategia consiste en diversificar sus fuentes de ingresos, mediante el incremento en la oferta de productos. Por su parte, la costumbre del mercado local de utilizar predominantemente el cemento en las edificaciones permite consolidar el posicionamiento de las cementeras".

- "Sin embargo, la fuerte competencia generada por la producción informal de productos no aptos para la construcción presiona a la baja los precios del cemento, perjudicando los márgenes de utilidad".

- "Asimismo, los elevados costos de transporte reducen la posibilidad de diversifi-
Keywords: Competitiveness. Investment and Financing Structure of Economic Value Added.

car las fuentes de ingresos mediante exportaciones, y las empresas cementeras aún se encuentran bastante concentradas en pocos mercados y productos".

Veamos otra opinión sobre el sector: ${ }^{3}$

"El monopolio que de hecho existía en el país en el sector cemento, centralizado en la región capital, fue roto con la formación de dos empresas privadas descentralizadas, Cementos Pacasmayo S.A., en 1957, y Cemento Andino S.A., en 1958. Posteriormente, la empresa capitalina instaló una pequeña planta en la localidad de Juliaca, que inició la producción en 1963, denominada en la actualidad Cemento Sur S.A. y en 1956 se crea la fábrica de Cemento Yura S.A. en Arequipa”.

"El total de la capacidad instalada en el país es de 3’460,000 TM/A de cemento, lo que significa una disposición de $163 \mathrm{Kg}$. de cemento por habitante. El Perú ocupa el sexto lugar en la producción de cemento en Latinoamérica luego de México, Brasil, Argentina, Colombia y Venezuela”.

Otros comentarios adicionales del sector: ${ }^{4}$

"Es importante mencionar que en enero 2011, el Gobierno tomó la decisión de reducir de 12 a $0 \%$ el arancel para cemento y clinker, con el objetivo de que se genere una reducción en los precios del mercado

2 Daniel Córdova. "La industria de cemento en el Perú: Favorables perspectivas de crecimiento en el largo plazo". Banco Wiese Ltdo. 17.02.2005. Lima. Perú.

3 Manuel Gonzales de la Cotera. "La industria del cemento en el Perú." p.1

4 Sergio Castro Deza. Informe trimestral. Apoyo \& Asociados. p.4 
interno. Sin embargo, cuando dicha medida estuvo vigente entre octubre 2007 y marzo 2010, los precios no registraron variaciones significativas. Además, las empresas del sector consideran que la medida no tendrá un impacto significativo en los precios, pues la demanda de cemento ha mantenido un fuerte crecimiento y porque los precios nacionales todavía resultan bastante competitivos”.

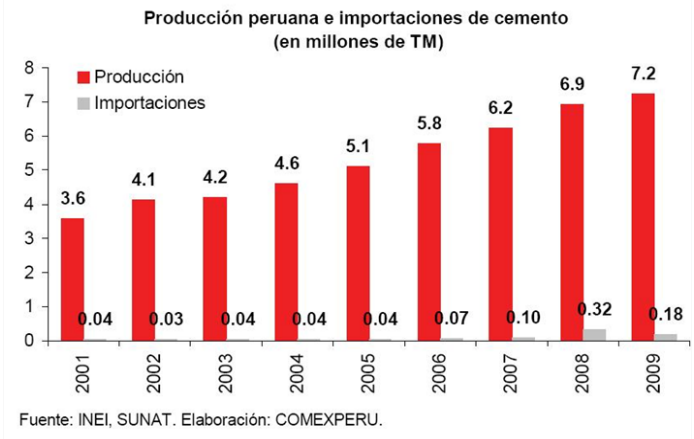

Pero analicemos en detalle desde la visión de las Empresas Calificadoras de Riesgo:

\section{Cemento Andino S.A.}

Según Equilibrium Clasificadora de Riesgos S.A ${ }^{5}$, expresa lo siguiente sobre esta empresa peruana:

"Su clasificación es AA.pe. La decisión se sustenta en el importante posicionamiento ostentado por la Empresa dentro de la región central del país, la adecuada solvencia de los principales accionistas y su reconocida experiencia en el rubro cementero. Del mismo modo se contemplan las inversiones que se vienen realizando en la ampliación de la capacidad productiva de planta, las cuales una vez concluidas se espera permitan fortalecer los niveles tanto de producción como de eficiencia”.

"La clasificación toma en consideración las características y condiciones de las emisiones que cuentan con resguardos financieros, los cuales comprometen a Cemento Andino a mantener ciertos indicadores financieros dentro de rangos que preserven la solidez financiera habiendo cumplido a la fecha de esta evaluación con todos los resguardos".

\section{Cementos Lima. S.A.}

Según Class \& Asociados S.A ${ }^{6}$, expresa a continuación lo que sigue:

"Su clasificación es AAA.pe y se sustenta en los siguientes factores:

- "Su liderazgo en el mercado cementero nacional, tanto en términos de volumen de producción, como en cuanto a los avances técnicos con los que opera, considerando que se trata de un sector con alto nivel tecnológico y con un elevado margen de capitalización".

- "Su participación en la construcción y en la administración de una planta productora de cemento en EE. UU de N.A., con lo cual controlará un acceso a este importante mercado, así como confirma el grado de calidad de su administración".

- "Su sólida situación financiera, así como su estabilidad administrativa, su importante capacidad de producción y los niveles de eficiencia operativa que ha alcanzado respaldados por una cartera de importantes proyectos de inversión en ejecución y por desarrollar".

- "La importante capacidad histórica de cobertura de deuda, el adecuado comportamiento mantenido en cuanto a la amortización puntual de su deuda contratada y acceso al crédito a adecuadas tasas de interés".

- "Las perspectivas positivas del sector construcción en el país”.

5 José Alonso Lucar. Equilibrium Clasificadora de Riesgo S.A., en un Informe de 8 páginas fechado al 10/01/2012. Lima. Perú.

6 Jeannette Valdiviezo. Class \& Asociados S.A., en un Informe de 11 páginas fechado al 30/09/2011. Lima. Perú. 
Cementos Pacasmayo S.A.A.

Según Apoyo \& Asociados ${ }^{7}$, especifica lo siguiente:

"Su clasificación es A1.pe y se sustenta en los siguientes factores:

1.- "La generación de flujos y los indicadores de cobertura: Durante los últimos 12 meses terminados en setiembre 2011, CPSAA registró un EBITDA (no incluye otros ingresos y egresos) de S/. 215.8 millones y un margen EBITDA de 33.3\% (S/. 228.6 millones y $36.7 \%$, respectivamente, a diciembre 2010)".

2.- "El sólido perfil de la Compañía y su posición como una de las mayores empresas productoras y comercializadoras de cemento del país. En este sentido, se debe destacar la estrategia de Cementos Pacasmayo consistente en la realización de una continua modernización y ampliación de sus instalaciones productivas y una diversificación de sus productos”.

3.- "La capacidad instalada y su posición de mercado: CPSAA cuenta con una significativa participación de mercado en el norte del país, lo cual se explica, entre otros, por la localización geográfica de su planta y su red de distribución. A ello se suman las barreras naturales para la entrada de nuevos competidores en la industria local del cemento. Asimismo, CPSAA continúa ampliando su capacidad instalada, lo que le permitirá responder a la mayor demanda generada por el sector".

\section{Cementos Yura S.A.}

Según Class \& Asociados S.A ${ }^{8}$, expresa a continuación lo que sigue:
"Su clasificación es AA+ y se sustenta en los siguientes factores:

- "El respaldo del Grupo al que pertenece, pues el ser parte del Grupo Gloria permite alcanzar un importante nivel de integración en procesos productivos relacionados, y contar con economías de escala, además de un importante esquema de gerencia corporativa".

- "El sector construcción es un impulsor del crecimiento económico del país, estando considerado como el sector más dinámico de los últimos años”.

- "Cuenta con una adecuada estructura operativa, apoyada por una gestión de procesos certificada de acuerdo a estándares internacionales, con una planta de producción en constante modernización y ampliación, determinando la generación de procesos productivos eficientes".

- "La empresa registra un adecuado nivel de deuda estructural, con un esquema de apalancamiento razonable para sus requerimientos financieros y operativos".

"Las perspectivas empresariales de Yura se presentan estables en razón a:

1) "Las proyecciones de ventas y la factibilidad de los proyectos de inversión destinados a ampliar la capacidad de su planta de producción";

2) "la adecuada estructura operativa y financiera, que permite una amplia capacidad de generación de fondos para cubrir sus obligaciones";

3) "los positivos y adecuados indicadores financieros"; $y$

4) "la trayectoria del Grupo Gloria, su solvencia patrimonial y su estructura corporati-va que ha integrado horizontalmente sus operaciones".

7 Sergio Castro Deza. Informe trimestral. Apoyo \& Asociados. Fechado a Febrero 2012.Lima. Perú.

8 Gisella del Águila. Class \& Asociados S.A., en un Informe de 13 páginas fechado al 30/09/2011. Lima. Perú. 
Este sector económico es actualmente el más destacado en la economía nacional, con un crecimiento en el 2010 de $17.4 \%$ y proyección total para el ejercicio de 2011 de cerca de 4\%, impulsado por la actividad privada, el cual es el motor de la expansión del sector, a través del desarrollo de viviendas multifamiliares, centros comerciales, supermercados y hoteles, así como por la ejecución de proyectos asociados a los sectores minero y energético, tanto en Lima, como en provincias. A ello se agrega la mayor inversión pública, que incluye la construcción y la rehabilitación de carreteras, de autopistas, de hospitales, de colegios, entre otras obras en desarrollo y en ejecución.

\section{ENFOQUE}

Perú fue la estrella económica de América Latina en la primera década del siglo XXI. En ese periodo, la inversión llegó a que el PBI (Producto Bruto Interno) per cápita registrase un crecimiento del $42 \%$. Lo que se tradujo en una mayor generación de empleo $y$, consecuentemente, en una reducción significativa de la pobreza, la que disminuyó de $55 \%$ en el 2000 a $31 \%$ en el 2010 . Avance que permitió sacar de la pobreza a más de cinco millones de peruanos. Nunca antes se había registrado en la historia del Perú que el ingreso se incrementara a tal velocidad y que la pobreza se redujese tanto en un periodo tan corto.
Por consiguiente, consideramos que es de interés nacional conocer cuán competitiva es la industria del cemento, materia prima básica de desarrollo de los proyectos de inversión, tanto en disponibilidad de capacidad instalada para producirlo, como en inventario del producto para atender al déficit de infraestructura de uso público, que alcanza a los 30 mil millones de dólares. Así, los conflictos sociales se originarían en parte por la carencia de aquella, que limita las inversiones y la creación de fuentes de empleo en el interior del país, donde se necesita mayor presencia del sector privado. Con el agravante de la pérdida anual de 250,000 peruanos que migran hacia el exterior en busca de mejores oportunidades de desarrollo personal.

Según la Asociación para el Fomento de la Infraestructura Nacional (AFIN), gremio empresarial que agrupa a las principales empresas privadas peruanas de infraestructura de servicios públicos en los sectores de energía, infraestructura de transportes, telecomunicaciones y saneamiento, explica en un amplio informe publicado en el diario Gestión de fecha 01.02.2012 ${ }^{9}$, que se está dejando de lado proyectos de inversión en asociación público-privado que tenía esa entidad, para concesionar bienes y servicios por US\$ 14,000 millones. Donde fundamentalmente se utilizará el cemento. 


\begin{tabular}{|l|c|c|}
\hline \multicolumn{1}{|c|}{ PROYECTOS NO CONSIDERADOS POR PROINVERSIÓN PARA EL ANO 2012 } \\
\hline \multicolumn{1}{|c|}{ PROYETOS } & SECTOR & $\begin{array}{c}\text { US\$ MILONES } \\
\text { SIN IGV. }\end{array}$ \\
\hline Aeropuerto chinchero & Aeropuertos & 356 \\
\hline Línea de transmisión Trujillo-Chiclayo & Energía & 110 \\
\hline Sistema de distribución de gas natural para el sur & Energía & 100 \\
\hline $\begin{array}{l}\text { Proyecto Especial Chavimochic Fase 1. Primera } \\
\text { etapa }\end{array}$ & Energía & 424 \\
\hline Afianzamiento hídrico cuenca del río Pisco-Rio Seco & Irrigación & 297 \\
\hline Proyecto Choclococha Desarrollado & Irrigación & 110 \\
\hline Puerto San Martín. Ica. & Puertos & 93 \\
\hline Puerto de Iquitos & Puertos & 22 \\
\hline Puerto de San Juan de Marcona & Puertos & 118 \\
\hline Navegabilidad de rutas fluviales Yurimaguas-Iquitos & Puertos & 46 \\
\hline Planta de desalinización de agua de mar en Lima Sur. & Saneamiento & 160 \\
\hline Carreteras IIRSA en tramos que definirá MTC & Transporte Terrestre & 776 \\
\hline Túnel trasandino ferroviario en Junín & Transporte Terrestre & 4,000 \\
\hline Longitudinal de la Selva & Transporte Terrestre & 3,000 \\
\hline Longitudinal de la Sierra & Transporte Terrestre & 4,200 \\
\hline $\begin{array}{l}\text { Banda ancha y masificación de fibra óptica en zonas } \\
\text { rurales }\end{array}$ & Telecomunicaciones & $\mathbf{1 4 , 0 1 2}$ \\
\hline & TOTAL & \\
\hline
\end{tabular}

Fuente: AFIN. Publicado en el diario Gestión de fecha 01.02.2012. Lima. Perú

¿Qué alternativas plantea esta situación creada por el explosivo desarrollo nacional?

a)Disponer toneladas de cemento, ladrillos, vigas de hierro, negro de humo, etc., para atender a la futura demanda de la construcción en el Perú.

b)Necesidad de contar con profesionales expertos en Proyectos de Inversión por parte del Gobierno, para aprobar con mayor celeridad los proyectos de inversión solicitados.

c) Oportunidad para crear trabajo masivo en el Perú, en un entorno de crisis mundial.

d)La desaceleración de las inversiones podría ahuyentar a los inversionistas que más bien deberíamos atraerlos considerando que la crisis mundial los ha alejado de los grandes países que enfrentan ese problema.

Sólo nos hemos orientado al análisis del pun- to a), si las cementeras nacionales tienen la capacidad para atender a la demanda interna en el corto y largo plazo con eficiencia y productividad generando valor económico agregado positivo para sus accionistas.

\section{RELACIÓN DE LA PROBLEMÁTICA ABORDADA CON EL TEMA CENTRAL DE LA XIII ASAMBLEA}

El tema central de ALAFEC, es: "Universidad y Sociedad: Técnica vs Humanismo”. Nuestro trabajo denominado " $¿ E s$ competitiva la Industria Cementera Peruana?", que ubicamos en Finanzas dentro de la subárea Modelos de Gestión Financiera, está en íntima conexión con el objetivo tres de la Conferencia: "Promover la investigación en los 
campos de la contaduría y la administración". Porque nuestra Facultad de Ciencias Contables, por intermedio de su Unidad de Posgrado, no sólo está en capacidad de actualizar conocimientos a funcionarios, públicos y privados, en temas de formulación y evaluación de proyectos de inversión, sino también, de asesorar directamente en la solución del cuello de botella en que evidentemente se encuentra el Gobierno actual para acelerar la autorización de las inversiones en el Perú y/o mejorar la productividad de la empresa privada buscando generar más utilidades para incrementar los impuestos fiscales $y$ coadyuvar al pleno empleo.

\section{METODOLOGÍA}

- Inductivo, porque analiza lo particular de las empresas industriales y las proyecta al conjunto empresarial materia de estudio.

- Deductivo, porque partiendo de las teorías y enfoques del costo estratégico arri ba a conclusiones específicas.

- De Síntesis, para construir la relación causa-efecto entre los objetos que componen los elementos de la investigación.

- De Análisis Documental, porque nos permitirá obtener la información principalmente de los estados financieros com- parados publicados por la Bolsa de Valores de Lima e informes individualizados de las empresas Clasificadoras de Riesgo como: Apoyo y Asociados; Class \& Asociados S.A.; Equilibrium Clasificadora de Riesgo S.A.

\section{MUESTRA}

Estudia la población completa de los consumidores del Perú por intermedio de las siguientes empresas cementeras distribuidas estratégicamente en todo el país:

\begin{tabular}{|l|l|}
\hline \multicolumn{1}{|c|}{ EMPRESAS } & \multicolumn{1}{c|}{ ZONA DE INFLUENCIA } \\
\hline Cemento Andino & $\begin{array}{l}\text { Sierra Central, Selva y parte de la } \\
\text { Región Lima. }\end{array}$ \\
\hline Cementos Lima & Lima. Capital del Perú. \\
\hline $\begin{array}{l}\text { Cementos Pacas- } \\
\text { mayo }\end{array}$ & $\begin{array}{l}\text { Chiclayo, Trujillo, Piura y Caja- } \\
\text { marca. }\end{array}$ \\
\hline Cementos Yura & $\begin{array}{l}\text { Arequipa, Cuzco, Tacna y Moque- } \\
\text { gua. }\end{array}$ \\
\hline
\end{tabular}

\section{RESULTADOS Y DISCUSIÒN. ANÁLISIS ESTRUCTURAL DE INVER- SIÓN Y FINANCIAMIENTO DE LA INDUSTRIA CEMENTERA}

A continuación procederemos al análisis del Balance General de las empresas del sector para determinar dos situaciones:

- La estructura de inversión y

- Forma de financiamiento de esa inversión.

CEMENTO ANDINO S.A.A. (Expresado en miles de nuevos soles) CUADRO No. 1. ANÁLISIS ESTRUCTURAL DE INVERSIÓN Y FINANCIAMIENTO

\begin{tabular}{|c|c|c|c|c|c|c|}
\hline \multirow{2}{*}{ CONCEPTO } & \multicolumn{2}{|c|}{2011} & \multicolumn{2}{|c|}{2010} & \multicolumn{2}{|c|}{ VARIACIÓN } \\
\hline & VALORES & $(\%)$ & VALORES & $(\%)$ & VALORES & $(\%)$ \\
\hline \multicolumn{7}{|c|}{ INVERSIONES } \\
\hline Activo Corriente & 197,342 & 10.89 & 193,370 & 12.13 & 3,972 & 1.82 \\
\hline Activo No Corriente & $1,615,567$ & 89.11 & $1,400,790$ & 87.87 & 214,777 & 98.18 \\
\hline TOTAL & $1,812,909$ & 100.00 & $1,594,160$ & 100.00 & 218,749 & 100.00 \\
\hline \multicolumn{7}{|c|}{ FINANCIAMIENTO } \\
\hline Capital Ajeno & 754,110 & 41,60 & 598,477 & 37.54 & 155,633 & 71.15 \\
\hline Capital Propio & $1,058,799$ & 58,40 & 995,683 & 62.46 & 63,116 & 28.85 \\
\hline TOTAL & $1,812,909$ & 100.00 & $1,594,160$ & 100.00 & 218,749 & 100.00 \\
\hline
\end{tabular}

Fuente: Bolsa de Valores de Lima. Estados Financieros Comparados. Año 2011-2010. Lima. Perú. 


\section{COMENTARIOS}

- El análisis de estructura de inversiones nos muestra una empresa típicamente industrial que prioriza el largo plazo en vez del corto plazo. (89\% el 2011 y $88 \%$ el 2010).

- La mayor inversión, por más de 218 millones de nuevos soles, se financió con el $71 \%$ de capital ajeno. Que se vio fortalecido en el año corriente al aumentar su posición del $37.54 \%$ al $41.60 \%$. Mientras que en la misma proporción disminuía el capital propio al caer de $62.46 \%$ en 2010 , a $58.40 \%$ en 2011. Cuyo efecto notable explica el mayor incre- mento de gastos financieros en más de 2 millones de nuevos soles. Es una deficiencia en el manejo financiero.

- Otra debilidad en el manejo operativo está en que la empresa a pesar de haber aumentado sus ventas en más de $2.5 \%$, respecto de 2010, sin embargo su ganancia bruta operativa disminuyó de $37.93 \%$ a $33.05 \%$ en el periodo bajo análisis.

- En consecuencia, la empresa ha visto disminuir su competitividad y ha generado una rentabilidad equivalente al $8,23 \%$ de su patrimonio contra el $8.99 \%$ del año previo.

CEMENTOS LIMA S.A.A. (Expresado en miles de nuevos soles)

CUADRO No. 2. ANÁLISIS ESTRUCTURAL DE INVERSIÓN Y FINANCIAMIENTO

\begin{tabular}{|l|l|l|l|l|l|l|}
\hline \multirow{2}{*}{ CONCEPTO } & \multicolumn{2}{c}{2011} & \multicolumn{2}{c|}{$\mathbf{2 0 1 0}$} & \multicolumn{2}{c|}{ VARIACIÓN } \\
\cline { 2 - 7 } & VALORES & $\mathbf{( \% )}$ & VALORES & $\mathbf{( \% )}$ & VALORES & $(\%)$ \\
\hline \multicolumn{7}{|c|}{ INVERSIONES } \\
\hline Activo Corriente & 396,042 & 10.37 & 571,746 & 15.20 & $(175,704)$ & $(306.51)$ \\
\hline Activo No Corriente & $3,423,211$ & 89.63 & $3,190,182$ & 84.80 & 233,029 & 98.18 \\
\hline TOTAL & $\mathbf{3 , 8 1 9 , 2 5 3}$ & $\mathbf{1 0 0 . 0 0}$ & $\mathbf{3 , 7 6 1 , 9 2 8}$ & $\mathbf{1 0 0 . 0 0}$ & $\mathbf{5 7 , 3 2 5}$ & $\mathbf{4 0 6 . 5 1}$ \\
\hline \multicolumn{7}{|c|}{ FINANCIAMIENTO } \\
\hline Capital Ajeno & $1,854,501$ & 48.56 & $1,960,470$ & 52,11 & $(105,969)$ & $(184.86)$ \\
\hline Capital Propio & $1,964,752$ & 51.44 & $1,801,458$ & 47.89 & 163,294 & 284.86 \\
\hline TOTAL & $\mathbf{3 , 8 1 9 , 2 5 3}$ & $\mathbf{1 0 0 . 0 0}$ & $\mathbf{3 , 7 6 1 , 9 2 8}$ & $\mathbf{1 0 0 . 0 0}$ & $\mathbf{5 7 , 3 2 5}$ & $\mathbf{1 0 0 . 0 0}$ \\
\hline
\end{tabular}

Fuente: Bolsa de Valores de Lima. Estados Financieros Comparados. Año 2011-2010. Lima. Perú.

\section{COMENTARIOS}

- El análisis de estructura de inversión nos muestra una empresa industrial que prioriza el largo plazo en vez del corto. (90\% el 2011 y $85 \%$ el 2010).

- La mayor inversión, por más de 57 millones de nuevos soles, se financió con el capital propio. Que se vio fortalecido en el año corriente al aumentar su posición del $47.89 \%$ a $51.44 \%$. Mientras que en la misma proporción disminuía el capital ajeno al caer de $52.11 \%$ en 2010 , a $48.56 \%$ en 2011 . Cuyo efecto notable explica el mayor incremento de ingresos financieros de más de 2 millones de nuevos soles. Muestra un manejo financiero eficiente porque recupera su autonomía financiera para tomar decisiones.

- Otra deficiencia encontrada está en que la empresa al término del año pese a que disminuyeron sus ventas en el 2011 en menos del $1 \%$ mostró una rentabilidad patrimonial del $11.26 \%$ contra $12.30 \%$ del año anterior.

- En consecuencia, la empresa ha visto mantener su competitividad porque ha mejorado su autonomía en la toma de decisiones pues la composición del capital propio es mayor que el ajeno en términos relativos y asegura a futuro la estabilidad financiera organizacional. 
CEMENTOS PACASMAYO S.A.A. (Expresado en miles de nuevos soles) CUADRO No. $3 . \quad$ ANÁLISIS ESTRUCTURAL DE INVERSIÓN Y FINANCIAMIENTO

\begin{tabular}{|l|l|l|l|l|l|l|}
\hline \multirow{2}{*}{ CONCEPTO } & \multicolumn{2}{c|}{2011} & \multicolumn{2}{c|}{2010} & \multicolumn{2}{c|}{ VARIACIÓN } \\
\cline { 2 - 7 } & VALORES & $\mathbf{( \% )}$ & VALORES & $\mathbf{( \% )}$ & VALORES & $(\%)$ \\
\hline \multicolumn{7}{|c|}{ INVERSIONES } \\
\hline Activo Corriente & 593,233 & 34.96 & 272,654 & 19.88 & 320,579 & 98.66 \\
\hline Activo No Corriente & $1,103,455$ & 65.04 & $1,099,116$ & 80.12 & 4,339 & 1.34 \\
\hline TOTAL & $\mathbf{1 , 6 9 6 , 6 8 8}$ & $\mathbf{1 0 0 . 0 0}$ & $\mathbf{1 , 3 7 1 , 7 7 0}$ & $\mathbf{1 0 0 . 0 0}$ & $\mathbf{3 2 4 , 9 1 8}$ & $\mathbf{1 0 0 . 0 0}$ \\
\hline \multicolumn{7}{|c|}{ FINANCIAMIENTO } \\
\hline Capital Ajeno & 769,162 & 45.33 & 510,190 & 37.19 & 258,972 & 79.70 \\
\hline Capital Propio & 927,526 & 54.67 & 861,580 & 62.81 & 65,946 & 20.30 \\
\hline TOTAL & $\mathbf{1 , 6 9 6 , 6 8 8}$ & $\mathbf{1 0 0 . 0 0}$ & $\mathbf{1 , 3 7 1 , 7 7 0}$ & $\mathbf{1 0 0 . 0 0}$ & $\mathbf{3 2 4 , 9 1 8}$ & $\mathbf{1 0 0 . 0 0}$ \\
\hline
\end{tabular}

Fuente: Bolsa de Valores de Lima. Estados Financieros Comparados. Año 2011-2010. Lima. Perú.

\section{COMENTARIOS}

- El análisis estructural de inversiones nos presenta una empresa industrial que prioriza el largo plazo en vez del corto. (65\% el 2011 y $80 \%$ el 2010).

- La mayor inversión, por cerca de 325 millones de nuevos soles, se financió con el $80 \%$ de capital ajeno. Que en el año corriente mejoró su posición del $37.19 \%$ al $45.33 \%$. Mientras que en la misma proporción disminuyó el capital propio de $62.81 \%$ en 2010 , a $54.67 \%$ en 2011 . Cuyo efecto notable explica un mayor gasto financiero que pasó de 13,354 millones de nuevos soles a 18,467 en el 2011. Muestra un manejo financiero deficiente.
- Una fortaleza identificada está en que la empresa al término del año mostró una rentabilidad patrimonial del $17.58 \%$ contra $17.17 \%$ del año anterior, logrando superar sus ventas en 5\% más que el año previo.

- En consecuencia, la empresa ha visto mejorar su competitividad al mantener su autonomía en la toma de decisiones porque la composición del capital propio es mayor que el ajeno, y, además, cuenta con suficiente disponibilidad de capacidad instalada para atender la demanda futura del país.

CEMENTOS YURA S.A.A. (Expresado en miles de nuevos soles) CUADRO No. 4. ANÁLISIS ESTRUCTURAL DE INVERSIÓN Y FINANCIAMIENTO

\begin{tabular}{|c|c|c|c|c|c|c|}
\hline \multirow{2}{*}{ CONCEPTO } & \multicolumn{2}{|c|}{2011} & \multicolumn{2}{|c|}{2010} & \multicolumn{2}{|c|}{ VARIACIÓN } \\
\hline & VALORES & $(\%)$ & VALORES & $(\%)$ & VALORES & $(\%)$ \\
\hline \multicolumn{7}{|c|}{ INVERSIONES } \\
\hline Activo Corriente & 267,502 & 16.74 & 319,061 & 24.41 & $(51,559)$ & $(17.74)$ \\
\hline Activo No Corriente & $1,330,497$ & 83.26 & 988,282 & 75.59 & 342,215 & 117.74 \\
\hline TOTAL & $1,597,999$ & 100.00 & $1,307,343$ & 100.00 & 290,656 & 100.00 \\
\hline \multicolumn{7}{|c|}{ FINANCIAMIENTO } \\
\hline Capital Ajeno & 760,189 & 47.57 & 592,766 & 45.34 & 167,423 & 57.60 \\
\hline Capital Propio & 837,810 & 52.43 & 714,577 & 54.66 & 123,233 & 42.40 \\
\hline TOTAL & $1,597,999$ & 100.00 & $1,307,343$ & 100.00 & 290,656 & 100.00 \\
\hline
\end{tabular}

Fuente: Bolsa de Valores de Lima. Estados Financieros Comparados. Año 2011-2010. Lima. Perú. 


\section{COMENTARIOS:}

- El análisis estructural de inversiones nos presenta una empresa industrial que prioriza el largo plazo en vez del corto. ( $83 \%$ el 2011 y $76 \%$ el 2010 ).

- La mayor inversión, por más de 290 millones de nuevos soles, se financió con el $58 \%$ de capital ajeno. Que se vio fortalecido en el año corriente al aumentar su posición del $45.34 \%$ al $47.57 \%$. Mientras que en la misma proporción disminuía el capital propio al caer de $54.66 \%$ en 2010, a 52.43\% en 2011. Cuyo efecto notable explica el mayor incremento de gastos financieros cuando su costo ha pasando de 17,488 millones de nuevos soles a 23,005 en el 2011. Es una deficiencia en el manejo financiero.

- Sin embargo, está planificando convenientemente el incremento de la capacidad instalada en previsión de una futura demanda en la región donde opera.

- En consecuencia, la empresa ha visto disminuir su autonomía para toma de decisiones por la caída de su capital propio pero ha generado una rentabilidad equivalente al $27,88 \%$ de su patrimonio, contra un $20.95 \%$ del año anterior merced al incremento de ventas netas en $15.76 \%$.

\section{CÁlCULO DE VALOR ECONÓMICO AGREGADO}

La herramienta clave que utiliza la empresa global para identificar si los costos estratégicos se aplican adecuadamente en el desarrollo de las actividades, es el denominado Valor Económico Agregado (EVA, siglas en inglés). Porque empresa que no crea valor sencillamente está consumiendo su patrimonio y amenazando su sostenibilidad en el largo plazo. Con tal intención presentamos a continuación cuatro cuadros de análisis relacionados al EVA:

Cuadro No. 5. Costo de capital de las empresas cementeras que listan en la Bolsa de Valores de Lima, por el periodo terminado al $31 / 12 / 2010$

Cuadro No. 6. Costo de capital de las empresas cementeras que listan en la Bolsa de Valores de Lima, por el periodo terminado al $31 / 12 / 2011$.

Cuadro No. 7. Cálculo del Valor Económico Agregado de las empresas cementeras por el periodo terminado al 31/12/2010.

Cuadro No. 8. Cálculo del Valor Económico Agregado de las empresas cementeras por el periodo terminado al 31/12/2011.

Se ha desarrollado de acuerdo al siguiente modelo:

a) Se revisó los estados financieros de años 2010 y 2009: Balance General y Estado de Ganancias y Pérdidas.

b) La posición de recursos se calculó así: Activo Corriente menos Pasivo Corriente, igual a Capital Neto de Trabajo, más Activo Fijo Neto.

c) Se ha tomado en cuenta los siguientes parámetros:

- Periodo de análisis: Año 2011 y 2010.

- Costo de Capital TEA TAMN: 7\% para 2010-201 $1^{10}$.

- Fuente de Información: Bolsa de Valores de Lima.

- Base de Información: Estados Financieros Comparados 2011 y 2010.

- Sector Evaluado: Empresas Industriales Cementeras.

d) En los Cuadro No. 5 y 6 se calculó el Costo de Capital de acuerdo a lo descrito en el punto (b).

e) En los Cuadros No. 7 y 8 se estableció el Valor Económico Agregado.

14 Tasa Efectiva Anual. Tasa anual en Moneda Nacional. Se asume 7\% para créditos a corto y largo plazo 


\begin{tabular}{|l|c|c|c|c|c|c|}
\hline \multicolumn{7}{|c|}{ CUADRO No. 5. COSTO DE CAPITAL DE EMPRESAS CEMENTERAS AÑO 2010.} \\
(Expresado en miles de nuevos soles) \\
\hline $\begin{array}{c}\text { EMPRESAS } \\
\text { CEMENTERAS }\end{array}$ & $\begin{array}{c}\text { ACTIVO } \\
\text { CORRIEN- } \\
\text { TE }\end{array}$ & $\begin{array}{c}\text { PASIVO } \\
\text { CORRIEN- } \\
\text { TE }\end{array}$ & $\begin{array}{c}\text { CAPITAL } \\
\text { NETO DE } \\
\text { TRABAJO }\end{array}$ & $\begin{array}{c}\text { ACTIVO } \\
\text { FIJO } \\
\text { NETO }\end{array}$ & $\begin{array}{c}\text { TOTAL } \\
\text { RECURSOS }\end{array}$ & $\begin{array}{c}\text { COSTO } \\
\text { CAPITAL } \\
7 \%\end{array}$ \\
\hline Cemento Andino & 193,370 & $(128,442)$ & 64,928 & $1,400,790$ & $1,465,718$ & 102.600 \\
\hline Cementos Lima & 571,746 & $(712,633)$ & $(140,887)$ & $3,190,182$ & $3,049,295$ & 213,451 \\
\hline $\begin{array}{l}\text { Cementos Pacas- } \\
\text { mayo }\end{array}$ & 272,654 & $(217,217)$ & 55,437 & $1,099,116$ & $1,154,553$ & 80,818 \\
\hline Cementos Yura & 319,061 & $(293,671)$ & 25,390 & 988,282 & $1,013,672$ & 70,957 \\
\hline TOTAL & $\mathbf{1 , 3 5 6 , 8 3 1}$ & $\mathbf{( 1 , 3 5 1 , 9 6 3 )}$ & $\mathbf{4 , 8 6 8}$ & $\mathbf{6 , 6 7 8 , 3 7 0}$ & $\mathbf{6 , 6 8 3 , 2 3 8}$ & $\mathbf{4 6 7 , 8 2 6}$ \\
\hline
\end{tabular}

Fuente: Bolsa de Valores de Lima. Estados Financieros Comparados: Años 2011-2010. Lima. Perú

\begin{tabular}{|l|c|c|c|c|c|c|}
\hline \multicolumn{7}{|c|}{ CUADRO No. 6. COSTO DE CAPITAL DE EMPRESAS CEMENTERAS AÑO 2011.} \\
(Expresado en miles de nuevos soles) \\
\hline $\begin{array}{c}\text { EMPRESAS } \\
\text { CEMENTERAS }\end{array}$ & $\begin{array}{c}\text { ACTIVO } \\
\text { CORRIEN- } \\
\text { TE }\end{array}$ & $\begin{array}{c}\text { PASIVO } \\
\text { CORRIEN- } \\
\text { TE }\end{array}$ & $\begin{array}{c}\text { CAPITAL } \\
\text { NETO DE } \\
\text { TRABAJO }\end{array}$ & $\begin{array}{c}\text { ACTIVO } \\
\text { FIJO } \\
\text { NETO }\end{array}$ & $\begin{array}{c}\text { TOTAL } \\
\text { RECURSOS }\end{array}$ & $\begin{array}{c}\text { COSTO } \\
\text { CAPITAL } \\
7 \%\end{array}$ \\
\hline Cemento Andino & 197,342 & $(153,820)$ & 43,522 & $1,615,567$ & $1,659,089$ & 116,136 \\
\hline Cementos Lima & 396,042 & $(718,465)$ & $(322,423)$ & $3,423,211$ & $3,100,788$ & 217,055 \\
\hline $\begin{array}{l}\text { Cementos Pacas- } \\
\text { mayo }\end{array}$ & 593,233 & $(252,625)$ & 340,608 & $1,103,455$ & $1,444,063$ & 101,084 \\
\hline Cementos Yura & 267,502 & $(303,991)$ & $(36,489)$ & $1,330,497$ & $1,294,008$ & 90,581 \\
\hline TOTAL & $\mathbf{1 , 4 5 4 , 1 1 9}$ & $\mathbf{( 1 , 4 2 8 , 9 0 1 )}$ & $\mathbf{2 5 , 2 1 8}$ & $\mathbf{7 , 4 7 2 , 7 3 0}$ & $\mathbf{7 , 4 9 7 , 9 4 8}$ & $\mathbf{5 2 4 . 8 5 6}$ \\
\hline
\end{tabular}

Fuente: Bolsa de Valores de Lima. Estados Financieros Comparados: Años 2011-2010. Lima. Perú

\begin{tabular}{|c|c|c|c|c|c|c|}
\hline \multicolumn{7}{|c|}{$\begin{array}{l}\text { CUADRO No. 7. CÁLCULO DE VALOR ECONÓMICO AGREGADO DE EMPRESAS } \\
\text { CEMENTERAS AÑO } 2010 \text { (Expresado en miles de nuevos soles) }\end{array}$} \\
\hline $\begin{array}{l}\text { EMPRESAS } \\
\text { CEMENTERAS }\end{array}$ & U.O.A.I & I.R. & U.N.D.I. & $\begin{array}{l}\text { C.D.C. } \\
7 \%\end{array}$ & E.V.A. & (\%) \\
\hline Cemento Andino & 137,278 & $(39,266)$ & 98,012 & $(102,600)$ & $(4,588)$ & $(2.38)$ \\
\hline Cementos Lima & 336,069 & $(81,164)$ & 254,905 & $(213,451)$ & 41,454 & 21.57 \\
\hline Cementos Pacasmayo & 223,018 & $(68,109)$ & 154,909 & $(80,818)$ & 74,091 & 38.56 \\
\hline Cementos Yura & 215,220 & $(63,077)$ & 152,143 & $(70,957)$ & 81,186 & 42.25 \\
\hline TOTAL & 911,585 & $(251,616)$ & 659,969 & $(467,826)$ & 192,143 & 100.00 \\
\hline
\end{tabular}

Fuente: Bolsa de Valores de Lima. Estados Financieros Comparados: Años 2011-2010. Lima. Perú 
CUADRO No. 8. CÁLCULO DE VALOR ECONÓMICO AGREGADO DE EMPRESAS CEMENTERAS AÑO 2011 (Expresado en miles de nuevos soles)

\begin{tabular}{|l|c|c|c|c|c|c|}
\hline $\begin{array}{c}\text { EMPRESAS } \\
\text { CEMENTERAS }\end{array}$ & U.O.A.I & I.R. & U.N.D.I. & $\begin{array}{c}\text { C.D.C. } \\
\mathbf{7 \%}\end{array}$ & E.V.A. & (\%) \\
\hline Cemento Andino & 129,560 & $(36,215)$ & 93,345 & $(116,136)$ & $(22,791)$ & $(20.77)$ \\
\hline Cementos Lima & 327,184 & $(92,290)$ & 234,894 & $(217,055)$ & 17,839 & 16.26 \\
\hline Cementos Pacasmayo & 179,818 & $(38,276)$ & 141,542 & $(101,084)$ & 40,458 & 36.87 \\
\hline Cementos Yura & 229,971 & $(65,158)$ & 164,813 & $(90,581)$ & 74,232 & 67.64 \\
\hline TOTAL & $\mathbf{8 6 6 , 5 3 3}$ & $\mathbf{( 2 3 1 , 9 3 9 )}$ & $\mathbf{6 3 4 , 5 9 4}$ & $\mathbf{( 5 2 4 , 8 5 6 )}$ & $\mathbf{1 0 9 , 7 3 8}$ & $\mathbf{1 0 0 . 0 0}$ \\
\hline
\end{tabular}

Fuente: Bolsa de Valores de Lima. Estados Financieros Comparados: Años 2011-2010. Lima. Perú

U.O.A.I = Utilidad Operativa antes de Impuestos; I.R= Impuesto a la Renta. U.N:D.I.= Utilidad Neta después de Impuestos; C.D.C.= Costo de Capital; E.V.A.= Valor Económico Agregado.

\section{DISCUSIÓN}

1. Analizaremos a continuación si la industria cementera es competitiva y si hubo reducción de costos:

Primero. Sector cementero no aplica en su totalidad uso del financiamiento a costo cero. El empresario peruano prefiere la emisión de bonos corporativos porque teme cambiar la estructura de su junta general de accionistas. El bono aumenta su capital ajeno y a su junta de accionistas la deja intacta. Mientras que la emisión de acciones incrementa su capital propio. Estrategia que debe cambiar paulatinamente si nuestros empresarios aspiran a globalizar su empresa y proyectarse al mundo entero vía exportación de sus productos. Cemento Andino y Cementos Lima, que pertenecen al Grupo Rizo Patrón, tienen la misma política: adquisición de subsidiarias y aumento de capacidad instalada con bonos y leasing financiero.

Cementos Pacasmayo financió con bonos su aumento de capacidad instalada y se diferenció de la competencia, adquiriendo subsidiarias con emisión de acciones.

Cementos Yura, repitió la receta: financiar con bonos y leasing financiero el incremento de su capacidad instalada.

\section{CUADRO No. 9. COSTO POR USO DE CAPITAL AJENO EN FINANCIAMIENTO DE OPE-} RACIONES AÑO 2010. (Expresado en Miles de Nuevos Soles).

\begin{tabular}{|c|c|c|c|c|c|}
\hline EMPRESAS & $\begin{array}{c}\text { INGRESOS } \\
\text { FINANCIEROS }\end{array}$ & $\begin{array}{c}\text { COSTOS } \\
\text { FINANCIEROS }\end{array}$ & $\begin{array}{c}\text { COSTO } \\
\text { NETO }\end{array}$ & $\begin{array}{l}\text { ESCUDO } \\
\text { FISCAL }^{11}\end{array}$ & (\%) \\
\hline Cemento Andino & 1,017 & $(9,711)$ & $(8,694)$ & 2.608 & 12.69 \\
\hline Cementos Lima & 6,173 & $(52,007)$ & $(45,834)$ & 13,750 & 66,91 \\
\hline Cementos Pacasmayo & 4,522 & $(13,354)$ & $(8,832)$ & 2,650 & 12.90 \\
\hline Cementos Yura & 11,909 & $(17,048)$ & $(5,139)$ & 1,542 & 7.50 \\
\hline TOTAL & 23,621 & $(92,120)$ & $(68,499)$ & 20,550 & 100.00 \\
\hline
\end{tabular}

Fuente: Estados Financieros Combinados. Bolsa de Valores de Lima. Año 2010-20009. Lima. Perú.

11 Impuesto dejado de pagar por ineficiencias que es el $30 \%$ de $68,49=20,550$. 
Segundo. La ineficiencia de la industria cementera le costó al país el año 2010, más de 68 millones de nuevos soles netos, dejando de pagar impuestos por más de 20 millones. El más eficiente en este periodo fue Cementos Yura porque so- lamente dejó de pagar más de un millón de nuevos soles. Y el más ineficiente fue Cemento Lima con más de 13 millones de nuevos soles con impuestos dejados de pagar. (Ver cuadro adjunto No. 9).

\section{CUADRO No. 10. COSTO POR USO DE CAPITAL AJENO EN FINANCIAMIENTO DE OPERACIONES AÑO 2010. (Expresado en Miles de Nuevos Soles).}

\begin{tabular}{|c|c|c|c|c|c|}
\hline EMPRESAS & $\begin{array}{c}\text { INGRESOS } \\
\text { FINANCIEROS }\end{array}$ & $\begin{array}{c}\text { COSTOS } \\
\text { FINANCIEROS } \\
\end{array}$ & $\begin{array}{l}\text { COSTO } \\
\text { NETO } \\
\end{array}$ & $\begin{array}{l}\text { ESCUDO } \\
\text { FISCAL }^{12} \\
\end{array}$ & $(\%)$ \\
\hline Cemento Andino & 293 & $(11,769)$ & $(11,476)$ & 3,443 & 109.20 \\
\hline Cementos Lima & 9,918 & $(53,069)$ & $(43,151)$ & 12,945 & 410.56 \\
\hline Cementos Pacasmayo & 3,474 & $(18,467)$ & $(14,993)$ & 4,498 & 142.66 \\
\hline Cementos Yura & 82,115 & $(23,005)$ & 59,110 & $(17,733)$ & $(562.42)$ \\
\hline TOTAL & 95,800 & $(106,310)$ & $(10,510)$ & 3,153 & 100.00 \\
\hline
\end{tabular}

Fuente: Estados Financieros Combinados. Bolsa de Valores de Lima. Año 2010-20009. Lima. Perú.

Tercero. En el año 2011, la brecha de costos financieros continuó aumentando. Pero los ingresos financieros mejoraron notablemente. El costo neto de palanqueo exagerado de capital ajeno alcanzó la suma de más de 10 millones de nuevos soles. Pero solamente se perdió 3 millones de nuevos soles por impuesto a la renta dejados de generar para el Fisco. Una recuperación seis veces menos que el año anterior. (Ver Cuadro No. 10).

Cuarto. Es digno mencionar que en el año 2011 la empresa Yura aparece como la más eficiente respecto de las demás empresas porque sus ingresos financieros superan a sus gastos financieros, pese a que su capital propio cayó de $54.66 \%$ en el 2010 a $52.43 \%$ en el 2011. (Ver Cuadro No. 4, página 14 y Cuadro No. 10, página 17).

2. ¿La industria cementera se benefició con el auge de la construcción de viviendas?
Veamos los resultados del análisis en el Cuadro No. 11, página 18.

\section{COMENTARIO}

- Cemento Andino es la empresa menos rentable en términos de margen bruto operativo tanto en el 2011 y 2010. Su índice cayó de 37.93 a $33.05 \%$., con ventas incrementadas en $2.5 \%$. Cementos Yura es la más competitiva en este segmento, porque su rentabilidad bruta va de 54.11 a $51.46 \%$ en 2011 . Y su ventas crecieron en $15.76 \%$.

- $\mathrm{Al}$ analizar la rentabilidad del patrimonio neto, la tendencia se confirma. Cemento Andino es menos rentable con propensión declinante. Va de 8.99 a $8.23 \%$ en el 2011. Por exceso de palanqueo de capital ajeno. En cambio Cementos Yura aparece más competitiva y rentable que aprovechó exitosamente el boom de la construcción en el país, pues su rentabilidad se elevó de 20.95 a $27.88 \%$ en el 2011.

12 El Escudo Fiscal parte del principio: "cada sol de oro que gano pago impuestos del 30\%; por cada sol oro que pierdo recupero 30\% de impuestos." En este caso las empresas al generar mayores gastos dejan de pagar impuestos a la renta. 


\begin{tabular}{|c|c|c|c|c|c|}
\hline \multicolumn{6}{|c|}{ CUADRO No. 11 ANÁLISIS DEL ÍNDICE DE RENTABILIDAD } \\
\hline EMPRESAS & INDICE & \multicolumn{2}{|l|}{2011} & \multicolumn{2}{|l|}{2010} \\
\hline \multicolumn{6}{|c|}{ RENTABILIDAD DEL MARGEN BRUTO OPERATIVO } \\
\hline \multirow{2}{*}{ Cemento Andino } & Utilidad Bruta * 100 & $147,080^{*} 100$ & $(\%)$ & $164,607^{*} 100$ & $(\%)$ \\
\hline & Ventas Netas & 445,039 & 33.05 & 434,017 & 37.93 \\
\hline \multirow{2}{*}{ Cementos Lima } & Utilidad Bruta ${ }^{*} 100$ & $514,592 * 100$ & 48.16 & $530,120 * 100$ & 49.26 \\
\hline & Ventas Netas & $1,068,482$ & & $1,076,139$ & \\
\hline \multirow{2}{*}{ Cementos Pacasmayo } & Utilidad Bruta ${ }^{*} 100$ & $329,990 * 100$ & 50.25 & $326,530 * 100$ & 52.36 \\
\hline & Ventas Netas & 656,659 & & 623,677 & \\
\hline \multirow{2}{*}{ Cementos Yura } & Utilidad Bruta * 100 & $296,677^{*} 100$ & 51.46 & $269,477^{*} 100$ & 54.11 \\
\hline & Ventas Netas & 576,527 & & 498,015 & \\
\hline & & & & & \\
\hline \multicolumn{6}{|c|}{ RENTABILIDAD DEL PATRIMONIO NETO. } \\
\hline \multirow{2}{*}{ Cemento Andino } & Utilidad Neta ${ }^{*} 100$ & $87,116^{*} 100$ & 8.23 & $89,487^{*} 100$ & 8.99 \\
\hline & Patrimonio Neto & $1,058,799$ & & 995,683 & \\
\hline \multirow{2}{*}{ Cementos Lima } & Utilidad Neta ${ }^{*} 100$ & $221,265^{*} 100$ & 11.26 & $221,507^{*} 100$ & 12.30 \\
\hline & Patrimonio Neto & $1,964,752$ & & $1,801,458$ & \\
\hline \multirow{2}{*}{ Cementos Pacasmayo } & Utilidad Neta* 100 & $163,063^{*} 100$ & 17.58 & $147,935^{*} 100$ & 17.17 \\
\hline & Patrimonio Neto & 927,526 & & 861,580 & \\
\hline \multirow{2}{*}{ Cementos Yura } & Utilidad Neta *100 & $233,614^{*} 100$ & 27.88 & $149,707^{*} 100$ & 20.95 \\
\hline & Patrimonio Neto & 837,810 & & 714,577 & \\
\hline TOTAL U. NETAS & & 705,058 & & 608,636 & \\
\hline
\end{tabular}

Fuente: Estados Financieros Combinados. Bolsa de Valores de Lima. Año 2011-2010. Lima. Perú.

3.Finalmente evaluamos la prueba de desemen el análisis del Cuadros No. 12: Cálculo del peño en Valor Económico Agregado, basado Valor Económico Agregado:

\begin{tabular}{|l|c|c|c|c|c|c|}
\hline \multicolumn{7}{|c|}{ CUADRO No.12. EVALUACIÓN DE VALOR ECONÓMICO AGREGADO $2011^{13}$} \\
(Expresado en Miles de Nuevos Soles)
\end{tabular}

Fuente: Bolsa de Valores de Lima. Estados Financieros Comparados: Años 2011-2010. Lima. Perú

13 A la Tasa Efectiva Anual de 7\%. 


\section{COMENTARIO}

- Cementos Lima, que lidera el mercado nacional en la venta de cemento en un $39 \%$ del total, utiliza el $41.36 \%$ de los recursos del sector, pero su valor económico agregado alcanza al $16.26 \%$, en base a una TEA anual del $7 \%$. Lo que significa que no es más rentable el que vende más. Y además, tiene problemas de liquidez, porque es la única empresa en el sector que tiene al término del año 2011, capital neto de trabajo negativo y con tendencia creciente.

- La empresa que aparece con mayor valor agregado, con más de 74 millones de nuevos soles es la Empresa Yura SAA, que, con el $17.25 \%$ de los recursos, genera el 67.64\% del VEA del sector.

\section{CONCLUSIONES}

La importación de cemento es un negocio demasiado costoso por los fletes, así no existan aranceles. Sin embargo, su crecimiento exponencial la hace atractiva a la competencia internacional. En octubre de 2007 se intentó eliminar el arancel a la importación del $12 \%$. Y es desde esa fecha para adelante que esta industria despertó de su letargo, acelerando "a locas y a tontas" el incremento de capacidad instalada de sus plantas. Aquí nuestras conclusiones:

(1) La industria cementera peruana es competitiva en la medida que no ha incrementado el precio unitario de la bolsa de cemento; ontribuye al mejoramiento del producto bruto interno, generando mayores puestos de trabajo y colaborando al desarrollo de las industrias colaterales. Resulta deficiente, en especial, el líder (Cementos Lima SAA), que maneja el 39\% de las ventas mercado, con el $41.36 \%$ de los recursos del sector.

Sólo genera un $16.26 \%$ de VEA cementero, por lo elevado de sus costos financieros, por no priorizar el aumento de capital vía emisión de acciones, por tener capital neto de trabajo negativo, y por no contribuir a la reducción de costos.

(2) El sector bajo estudio se benefició con el auge de construcciones de viviendas porque sus utilidades netas después de impuestos entre 2010 al 2011, se incrementaron en 96,422 millones de nuevos soles, es decir, un aceptable 15.84\%. Sólo limitado por su ineficiencia en el manejo financiero cuyo costo por intereses saltó de 92 millones de nuevos soles en 2010, a más de 106 millones en 2011. Reduciendo ineficiencias en este periodo, impuestos dejados de pagar en el 2010 por más de 20 millones a solamente 3 millones en el 2011. (Ver cuadro No. $9 y$ 10, página 17). Aprovecharon la oportunidad para mejorar su productividad por la presencia en el mercado limeño de la competencia extranjera.

(3) La medida de desempeño de valor económico agregado para el sector, según análisis aparte, (Ver Cuadro No. 12, página 19), a una TEA de 7\%, en moneda nacional, fue positiva para todas las empresas cementeras, excepto Cemento Andino.

\section{RECOMENDACIONES}

(1) Empresas con dominio de mercado, como es el caso de la industria cementera, deben tener preferente atención 
de INDECOPI, identificando aquellas barreras y restricciones que han sobrevivido a la liberalización de los mercados, estimulando la inversión y la entrada de nuevos competidores en beneficio del bienestar de los consumidores. Organismos responsables de las políticas de competencia deben priorizar la supervisión de aquellas industrias altamente concentradas, que involucran costos hundidos elevados, y que están menos expuestas a la competencia internacional. Promoviendo la investigación y la prevención antes de acciones que limitan o distorsionan la competitividad. Incentivando así la cooperación ESTADOUNIVERSIDAD-EMPRESA.

(2) El tema financiamiento de inversiones de las sociedades anónimas abiertas que listan en la Bolsa de Valores de Lima, debe ser motivo de revisión del Reglamento de la Superintendencia del Mercado de Valores, en cuanto a la obligatoriedad de emitir acciones en vez de bonos. Valores que solamente deben utilizar los Gobiernos por razones de necesidad pública. $Y$ de una vez por todas promover el Mercado de Valores Peruano, que es pequeño y de poca profundidad, a través del accionariado difundido. Porque beneficia a la minoría accionaria, costos empresariales más bajos, contribuye a optimizar la rentabilidad, sin afectar la economía de los consumidores y de todos los Stakeholders14 que participan en un mercado abierto. En este sector en necesario promover las ventajas del buen gobierno corporativo.

(3) Los organismos superiores de control lámese Contraloría General de la Repú- blica, Superintendencia de Banca y Seguros, Contaduría Pública de la Nación, Superintendencia del Mercado de Valores, etc., en defensa del interés público, deben promover la aplicación obligatoria como medida de desempeño, el Valor Económico Agregado. Para su exigencia al final de cada año el Banco Central de Reserva debe publicar el costo promedio ponderado de la Tasa Efectiva Anual en moneda nacional y en moneda extranjera, para aplicar al cálculo del Valor Económico Agregado en forma obligatoria a nivel nacional.

\section{REFERENCIAS BIBLIOGRÁFICAS}

1. Bolsa de Valores de Lima.2012 Estados financieros comparados 2011-2010. Marzo. Lima. Perú.

2. Brealey, Richard. Myers, Steward. Principios de finanzas corporativas. Editora McGraw-Hill. México D.F. México.

3. Castro Deza, Sergio. 2011. Informe trimestral. Apoyo \& Asociados. Lima. Perú.

4. Castro Deza, Sergio. 2012. Informe Trimestral. Apoyo \& Asociados. Lima. Perú.

5. Córdova, Daniel. 2005. La industria de cemento en el Perú: Favorables perspectivas de crecimiento en el largo plazo. (Informe) Banco Wiese Ltdo. Lima. Perú.

6. Del Águila, Gisella. Class \& Asociados S.A., Informe de 13 páginas fechado al 30/09/2011. Lima. Perú.

7. García, Elías. "Ejecución de Proyectos en APP en 2012 será 50\% menor que en 2010”. Diario Gestión. Lima. Perú.

14 Es decir, cualquier persona o entidad que es afectada por las actividades de una organización; por ejemplo, los trabajadores de una organización, sus accionistas, las asociaciones de vecinos, sindicatos, organizaciones civiles y gubernamentales, etc. 
8. Gonzales de la Cotera, Manuel. 1998 La industria del cemento en el Perú. Lima. Perú.

9. Lucar, José Alonso. Equilibrium Clasificadora de Riesgo S.A. 10/01/2012. Informe. Lima. Perú.

10. Valdiviezo, Jeannette. Class \& Asociados S.A. 30/09/2011. Informe. Lima. Perú.
11. Organización Internacional del Trabajo. 2002 Guía para mejorar la productividad de la pequeña y la mediana empresa. Confederación de la Producción y del Comercio. Santiago de Chile. Chile.

12. Plan nacional de competitividad. 2005. "Informe del Consejo Nacional de la Competitividad”. Lima. Perú. 